\title{
A STUDY ON EFFECT OF BOWING ON A SCANS USING PULSE ECHO ULTRASONIC TECHNIQUE
}

\author{
Sujatha Kumaran ${ }^{1}$, Sheela Rani.B ${ }^{2}$ \\ ${ }^{1}$ Research Scholar, Department of Electronics \& Instrumentation, Sathyabama University, Chennai,Tamil Nadu, \\ India, ksujatha71@gmail.com \\ ${ }^{2}$ Vice-Chancellor, Sathyabama University, Chennai, Tamil Nadu, India, kavi_sheela@yahoo.com
}

\begin{abstract}
This paper deals with the study of A Scans obtained from sub assembly head top used in a Prototype Fast Breeder Reactor. A sub-assembly head top is the specimen used to determine the effect of bowing on A Scans. The condition of bowing has been simulated. The A Scans are obtained under normal conditions of the ring and also under simulated bowing conditions. The envelope of the A Scans under both the conditions have been compared. It is found that the two edges of the ring are clearly indicated through the A Scan. Under normal conditions, the two edges have a slight difference in the depth. This is due to the constructional feature of the object. But under simulated conditions, the two edges are obtained at two different depths, of magnitude higher than the normal conditions. The shift in the A Scans is clearly seen It is observed that greater the tilt given for simulated bowing, larger is the shift in the depth of the two edges of the ring.
\end{abstract}

Index Terms: Ultrasonic, Pulse Echo, Bowing, A Scans

\section{INTRODUCTION}

Pulse echo Ultrasonic Technique is one of the most commonly applied technique for determination of thickness, depth, and detection of flaws in material and estimation of its dimensions. The advantage of pulse Echo technique is that a single transducer performs the job of transmitting and receiving, hence only a single side access is required. The transducers can be of contact type or immersion type. The probe can be of normal beam or angled beam. In the pulse echo method, the transducer emits short bursts or pulses of ultrasound into the material. These waves get reflected from the top surface of the specimen, travel through the specimen and get reflected again at the back wall of the surface. In between if there is any flaw; the waves get reflected at the flaws. The reflected waves are called echoes, which are displayed in the form of A Scans, B Scans and C Scan. The A-scan represents the amount of ultrasonic energy received as a function of time. The B-scan presentations provide a cross-sectional view of the test specimen. The C-scan presentation provides a plan-type view of the location and size of test specimen features. Pulse echo methods include compression, shear and surface wave mode testing. In the compression wave mode, waves are sent into the material perpendicular to the specimen's surface. For this, the normal beam probes are used. In shear wave mode, waves are directed in an angle into the material using angled beam probes. While using contact type transducers, suitable couplants are used as medium between the transducer and the specimen. For immersion transducers, water is usually the medium. To obtain information about depth, the transducer is placed vertically above the object. To get information about thickness, the transducer is placed laterally to the specimen

Bowing is a common condition prevailing in nuclear reactors. Fuel channel bow is nothing but shifting of the fuel rod towards one side. One face of the fuel channel becomes elongated than the other face. Bowing can occur due to initial manufacturing, residual stress relaxation under irradiation, and differential irradiation growth. At the start of every fuel cycle, it is mandatory to check for these abnormal conditions. At IGCAR Kalpakkam, an ultrasonic undersodium scanner has been developed to image the subassembly tops for the detection of anomalies like bowing and growth.[1] A C-Scan image is obtained and software has been developed to indicate the depth variation in different colours. Thus bowing and growth can be easily detected.[2]

This paper involves the study of effect of bowing on A Scans obtained from the sub-assembly tops. The study involves applying pulse echo technique on the Sub assembly top. A pulse echo transducer is made to step with a spatial sampling length across the surface of the specimen, which is the sub-assembly top. [3-5]. The sub-assembly head is placed in an immersion tank and ultrasonic wave is directed into it. At every step, the pulser emits ultrasonic waves. These waves get reflected at the top surface of the object and echoes are obtained in the form of A Scans. The A Scan so received comprises of the transmitted wave, first echo which is the reflected wave from the interface and at the last the back wall echo. The back wall echo is the reflected wave from the bottom of the immersion tank. Due to the beam spread of the ultrasonic wave, the transducer starts detecting the object much before along the scan axis than when the transducer is actually above the object. 
According to the depth of reflection the echoes are positioned in A Scans, in the sense, echo reflected from the top surface of the specimen is seen first, and the back wall echo is seen at the end of A Scan. If the defect or flaw is closer to the top surface, this echo is closer to the first scan, on the other hand if the defect is nearer to the bottom surface of the specimen, then the echo is seen nearer to the back wall echo. The defect will always be seen between the first echo and the back wall echo. If there is more than one defect, then accordingly the number of echoes will also be more.

\section{DATA ACQUISITION}

A piezoelectric crystal of $5 \mathrm{MHz}$ frequency with a normal beam probe is used as the transducer. The transducer diameter is $6 \mathrm{~mm}$ and it is a focussed transducer. It is attached to the actuator through stepper motors. The stepper motor is controlled through software. The Pulser -Receiver used is Olympus model Parametrics 5900PR. It is capable of moving the transducer along 4 axes. The velocity of the transducer is set to $1 \mathrm{rpm}$, the step length is set to $0.5 \mathrm{~mm}$. The transducer is kept at a distance of $30 \mathrm{~mm}$ form the top surface of the object in the immersion tank. The sampling frequency is set to $100 \mathrm{MHz}$ and the recording length is set to 400. The object is the sub-assemble head top, which is shown in Fig.1. The object is $15 \mathrm{~cm}$ in height; the outer diameter is $12 \mathrm{~cm}$. The shell thickness is $3.2 \mathrm{~mm}$. The inner edge is slightly tapering in nature.

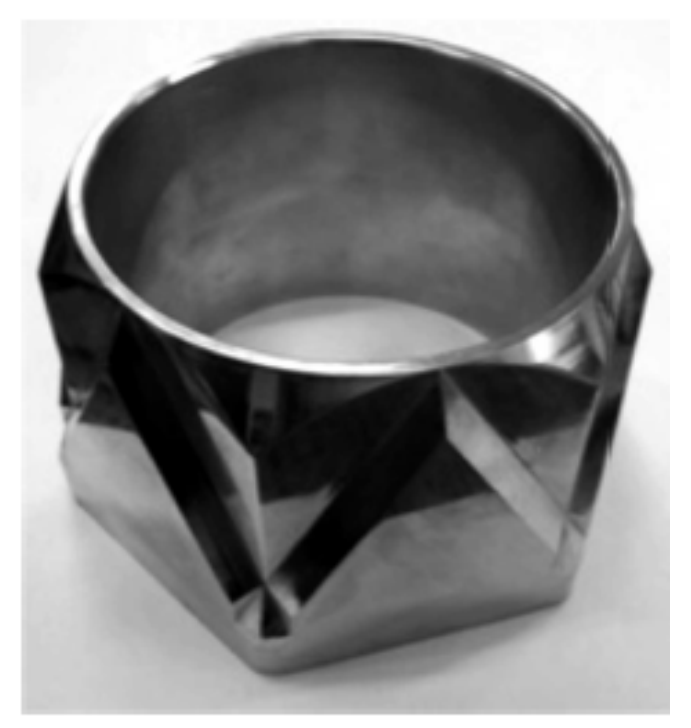

Fig -1: Object under study: Sub-assembly head

The transducer is placed along the horizontal axis where it just begins to detect the object. This is the place where the edge of the ultrasonic beam hits the object. The transducer emits pulses and the reflected echo is received, digitised and stored. The transducer is moved by $0.5 \mathrm{~mm}$ and the next $\mathrm{A}$ Scan is recorded. The transducer is moved till the edge of the ultrasonic beam hits the inner edge of the object. In other words, the transducer is moved till it stops detecting the object. The movement of the object is depicted in Fig.2. Thus 16 number of A Scans is recorded.

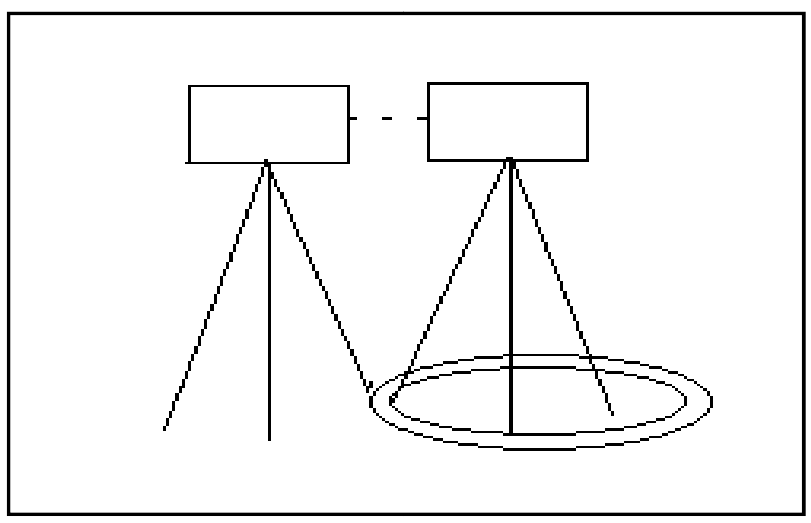

Fig -2: Movement of the transducer

To simulate bowing, one of the edges of the object is raised by placing an object of height $10 \mathrm{~mm}$ underneath that face of the sub-assembly head. This causes one of the faces of the object to be raised than the other face. Thus the subassembly head is tilted, which is equivalent to bowing. The vertical position of the transducer is maintained the same. The transducer is positioned along the horizontal axis where it just begins to detect the object. The transducer is moved by $0.5 \mathrm{~mm}$ and for every position an A Scan is recorded. This is continued till the transducer stops detecting the object.

\section{METHODOLOGY}

In each A Scan, only 400 samples are recorded. The trigger delay is set to 25 microseconds. Therefore the transmitted wave and the back wall echo are not recorded in this case. Only the echo obtained from the top surface of the object is recorded as shown in Fig.3.

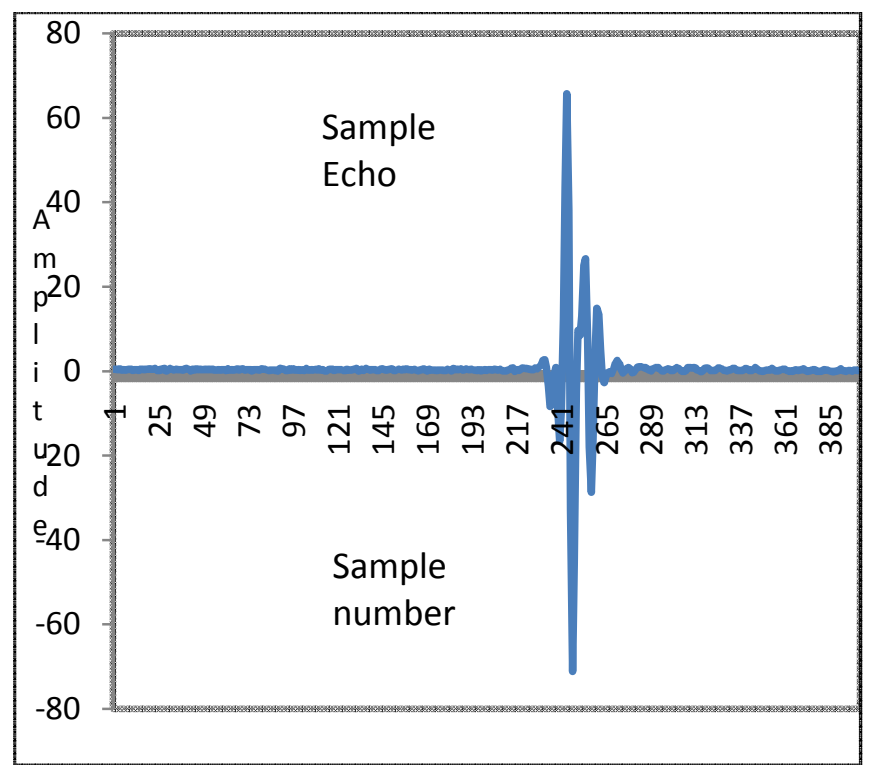

Fig -3: Sample Echo

The envelope of the A scan is determined using Hilbert transform. The echo obtained is a RF signal. In order to extract the envelope from the real-valued RF-echoes, a complex-valued signal is formed and then the envelope of 
the signal is obtained by taking the absolute value of that complex valued signal.

\section{RESULTS AND DISCUSSION}

The sub-assembly head of $3.2 \mathrm{~mm}$ width and $12 \mathrm{~cm}$ in outer diameter, $15 \mathrm{~cm}$ in height is the object kept in an immersion tank filled with water. The focused normal beam transducer is placed $30 \mathrm{~mm}$ above the object and A Scans are recorded for every $0.5 \mathrm{~mm}$ movement of the transducer. 16 A Scans are recorded and the envelope of few of the 16 A Scans recorded under normal conditions is shown in Fig.4

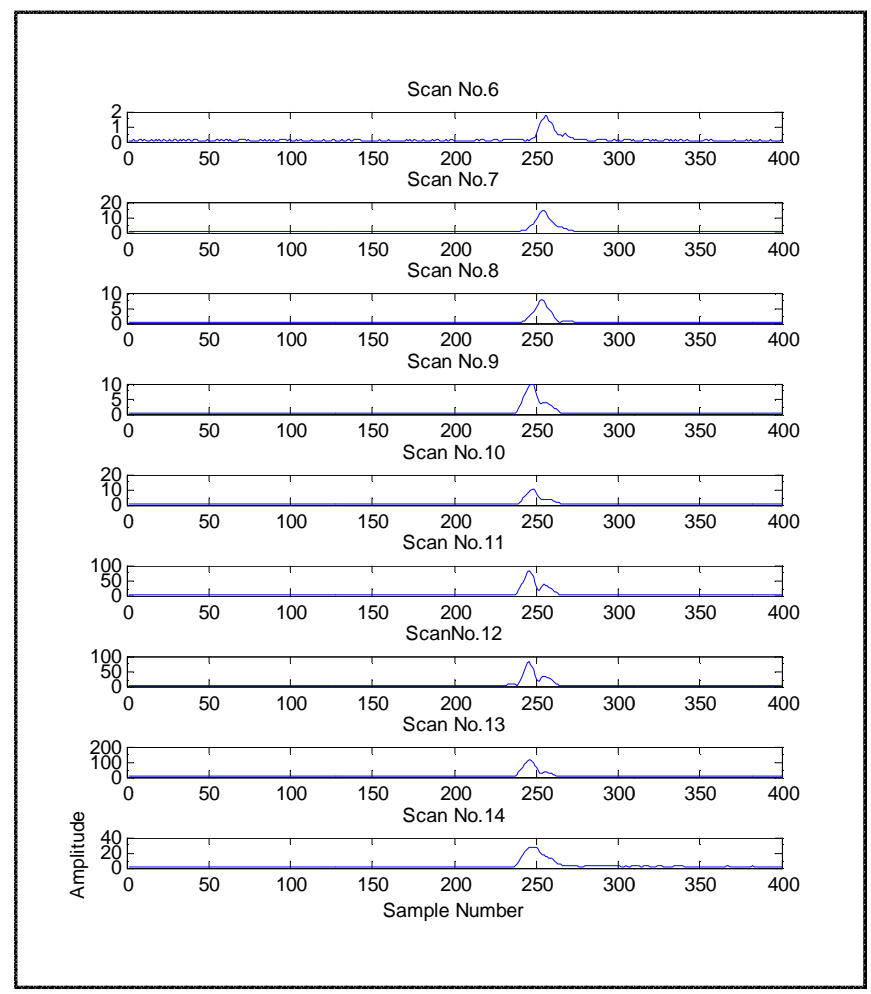

Fig -4: Envelope of A Scans under normal conditions

The horizontal axis of the above figure denotes the sample number and the vertical axis denotes amplitude of the echo. From the above figure, it is seen that the peak of the Scan No.6 is obtained at sample number 257. In Scan No.7, The amplitude of the peak has increased, indicating more of the ultrasonic beam hits the edge of the object. In Scan No.9, there are two peaks seen, which suggests that the transducer is able to see both the edges. In Scan No.11 and 12, both the edges are seen, which is seen by two peaks in the envelope. The amplitude of the peak has increased, indicating more of beam is facing the object. In Scan No.14, The peak occurs at sample number 247. The difference in the depth is due to the tapering inner edge of the A Scan. Thus to separately detect the two edges of the sub-assembly head, the transducer has moved a distance of $4 \mathrm{~mm}$.

The object is given a tilt on one side by elevating it by placing a $10 \mathrm{~mm}$ height piece below it. There are 13 A Scans recorded for start of detection and stopping of detection of the object. The envelopes of few of the A Scans recorded under simulated bowing conditions are shown in Fig.5.

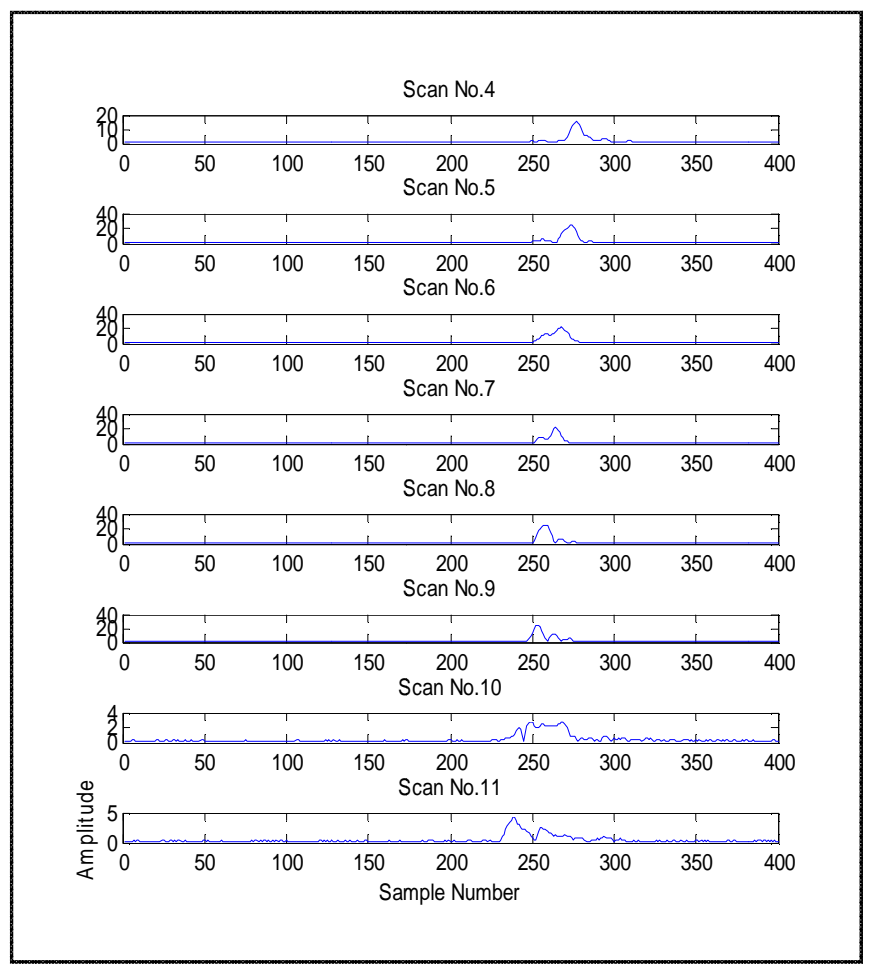

Fig -5: Envelope of A Scans under simulated bowing conditions

The peak in Scan No.4 is obtained at sample number 278. In this case also, in the next consecutive scans, two peaks are seen. Thus the transducer is able to see both the edges. The peak is seen to shift towards left. In Scan No.11, the peak is obtained at sample number 239. The two peaks are obtained at samples 278 and 239. This indicates that one edge is at different depth than the other. The difference in sample number was only 10 under normal conditions and under simulated bowing conditions; the difference in sample number is greater. The transducer has traversed a distance of $3 \mathrm{~mm}$ to cover both the edges. This is in agreement with the fact that the shell thickness of a bowed sub-assembly head top seems to reduce in size when seen from top.

\section{CONCLUSIONS}

The condition of bowing has been simulated. The A Scans recorded under normal conditions and simulated bowing conditions are compared. Both the edges of the object are clearly seen in the envelope of the A Scans obtained. Under normal conditions, the peak obtained due to the edges is almost at the same depth, the sample number differing by 10 only. Under simulated bowing conditions, the peaks obtained due to both the edges is at different depths. This indicates that one edge is at higher level than the other edge. This occurs during bowing. Thus the condition of bowing can be detected by means of A Scans. The extent of bowing can also be estimated by determining the separation of the two peaks and the sample numbers at which the peaks have occurred. Larger the gap between the two peaks more is the extent of bowing. Thus the condition of bowing can also be detected using A Scans, but can be done as a post- 
processing technique only. This technique cannot be applied to measure in-situ bowing.

\section{ACKNOWLEDGEMENT}

The authors wish to thank the scientists Dr.P.Kalyanasundaram, Dr.B.Venkatraman \& Mr.V.Prakash of Indira Gandhi Centre for Atomic Research, Government of India, for the technical support provided by them.

\section{REFERENCES}

[1]. Patankar V.H, Chaurasia Reetesh, Asokane.C, Gunasekaran.T.G, Varier Vijayan, Sylvia T.J, "Design and Development of Automated Ultrasonic Instrumentation for detection of growth and bowing of under-sodium fuel subassemblies of FBR" Proceedings of the National Seminar \& exhibition on Non-Destructiive Evaluation, NDE 2009, Dec10-12, 2009, pp 120-125.

[2].K.Swaminathan, C.Asokane, J.I.Sylvia, P.Kalyanasundaram, P.Swaminathan, "An Ultrasonic Technique Scanning Technique for In-Situ Bowing Maesurement of Prototype Fast Breeder Test Reactor", IEEE Transactions on Nuclear Science. Vo.59, No..1, February 2012.

[3]. O.Martinez, M. Parrilla, M. A. G. Izquierdo and L. G. Ullate (1999), "Application of digital signal processing techniques to synthetic aperture focusing technique images." Sensors and Actuators A: Physical Volume 76, Issues 1-3, 30 August 1999, pp 448-456.

[4]. L.Satyanarayan, Krishnan Balasubramaniam and C.V.Krishnamurthy, "Comparative Studies on MultiElement Synthetic Aperture Focusing Phased Array Ultrasonic Technique and Conventional SAFT", www.cndeiitm.net.

[5].Sujatha Kumaran, Sheela Rani.B, Manoharan.N , Kalyanasundaram,( 2008), " A Study on the Estimation of Width using Synthetic Aperture Focusing Technique.", Proceedings of International Conference on Emerging Scenarios in Space Technology and applications (ESSTA2008), Sathyabama University, Jeppiaar Nagar, Chennai, India.

\section{BIOGRAPHIES}

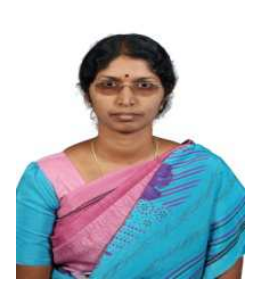

Sujatha Kumaran is working as a faculty in Department of Electronics \& Instrumentation, Sathyabama University, Chennai, India. She is also currently a research scholar and her area of research is Non- Destructive Evaluation using Ultrasonics.

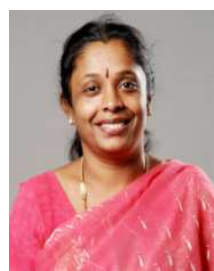

Dr.B.Sheela Rani completed M.S (By Research) from Anna University, Chennai(1999). She obtained her Doctorate Degree from Anna University, Chennai(2003). Currently, she is the Vice Chancellor of Sathyabama University, Chennai. She has published more than 150 papers in various Conferences and Journals. Her areas of research include Signal Processing, Image Processing and Soft Computing. 